\title{
Pacific
}

Journal of

Mathematics

\section{SOME NUMERIC RESULTS ON ROOT SYSTEMS}

\section{J. Y. SHI}




\section{SOME NUMERIC RESULTS ON ROOT SYSTEMS}

\section{JIAN-YI SHI}

Let $\Phi$ be an irreducible root system (sometimes we denote $\Phi$ by $\Phi(X)$ to indicate its type $X)$. Choose a simple root system $\Pi$ in $\Phi$. Let $\Phi^{+}$(resp. $\Phi^{-}$) be the corresponding positive (resp. negative) root system of $\Phi$. By a subsystem $\Phi^{\prime}$ of $\Phi$ (resp. of $\Phi^{+}$), we mean that $\Phi^{\prime}$ is a subset of $\Phi$ (resp. of $\Phi^{+}$) which itself forms a root system (resp. a positive root system). We refer the readers to Bourbaki's book for the detailed information about root systems. Among all subsystems of $\Phi$, the subsystems of $\Phi$ of rank 2 and of type $\neq A_{1} \times A_{1}$ are of particular importance in the theory of Weyl groups and affine Weyl groups (see the papers by Jian-yi Shi). In the present paper, we shall compute the number of such subsystems of $\Phi$ for an irreducible root system $\Phi$ of any type. Some interesting properties of $\Phi$ are also obtained.

1. The number $h(\alpha)$. Let $\langle$,$\rangle be an inner product of the euclidean$ space $E$ spanned by $\Phi$. For any $\alpha \in \Phi$, we denote by $|\alpha|$ the length of $\alpha$, by $\alpha^{\vee}$ the dual root $2 \alpha /\langle\alpha, \alpha\rangle$ of $\alpha$ and by $s_{\alpha}$ the reflection in $E$ which sends any vector $v \in E$ to $s_{\alpha}(v)=v-\left\langle v, \alpha^{\vee}\right\rangle \alpha$. For $\alpha, \beta \in \Phi$, we write $\alpha<\beta$ if $\beta-\alpha$ is a sum of some positive roots.

For $\alpha \in \Phi$, we define the sets $D(\alpha)=\{\beta \in \Phi \mid \alpha+\beta \in \Phi\}$, $D^{+}(\alpha)=D(\alpha) \cap \Phi^{+}$and $D^{-}(\alpha)=D(\alpha) \cap \Phi^{-}$. Let $d(\alpha)$ be the cardinality of the set $D^{+}(\alpha)$. Also, we denote by $h t(\alpha)$ the height of $\alpha$, i.e. $\operatorname{ht}(\alpha)=\sum_{\beta \in \Pi} a_{\beta}$ if $\alpha=\sum_{\beta \in \Pi} a_{\beta} \beta$ with $a_{\beta} \in \mathbb{Z}$.

For any $\alpha \in \Phi^{+}$, there exists a sequence $\xi$ of roots $\alpha_{1}=\alpha, \alpha_{2}, \ldots$, $\alpha_{r}$ in $\Phi^{+}$such that $\alpha_{r} \in \Pi$ and for every $i, 1<i \leq r$, we have $\alpha_{i-1}>\alpha_{i}=s_{\delta_{i}}\left(\alpha_{i-1}\right)$ for some $\delta_{i} \in \Pi$. Such a sequence $\xi$ is called a root path from $\alpha$ to $\Pi$. We denote by $h(\alpha, \xi)$ the length $r$ of $\xi$. We shall deduce a formula for the number $h(\alpha, \xi)$, from which we shall see that $h(\alpha, \xi)$ is actually independent on the choice of a root path $\xi$ from $\alpha$ to $\Pi$ but only dependent on the root $\alpha$.

Note that if the root system $\Phi$ contains roots of two different lengths and if $\alpha=\sum_{\beta \in \Pi} a_{\beta} \beta$ is a long root of $\Phi$ with $a_{\beta} \in \mathbb{Z}$ then each coefficient $a_{\beta}$ with $\beta$ short is divisible by $|\alpha|^{2} /|\beta|^{2}$.

LEMMA 1.1. Let $\alpha=\sum_{\beta \in \Pi} a_{\beta} \beta, a_{\beta} \in \mathbb{Z}$, be a root of $\Phi^{+}$and let $\xi$ be a root path from $\alpha$ to $\Pi$. Then 
(i) If either all the roots of $\Phi$ have the same length or $\alpha$ is a short root of $\Phi$ with $\Phi$ containing roots of two different lengths, then $h(\alpha, \xi)=\operatorname{ht}(\alpha)$;

(ii) If $\alpha$ is a long root of $\Phi$ with $\Phi$ containing roots of two different lengths, then

$$
h(x, \xi)=\sum_{\beta \in \Pi} \frac{|\beta|^{2}}{|\alpha|^{2}} a_{\beta} .
$$

Proof. Let $\alpha_{1}=\alpha, \alpha_{2}, \ldots, \alpha_{r}$ be a root path from $\alpha$ to $\Pi$. Then in case (i), we have $\operatorname{ht}\left(\alpha_{i}\right)=\mathrm{ht}\left(\alpha_{i+1}\right)+1$ for any $i, 1 \leq i<r$, by the fact that $\left\langle\alpha_{i}, \delta_{i}^{\vee}\right\rangle=1$, where $\delta_{i} \in \Pi$ satisfies the relation $\delta_{i}\left(\alpha_{i-1}\right)=$ $\alpha_{i}$. So assertion (i) follows immediately by applying induction on $\mathrm{ht}(\alpha) \geq 1$. Next assume that we are in case (ii). Again apply induction on $\operatorname{ht}(\alpha) \geq 1$. If $\operatorname{ht}(\alpha)=1$, then $\alpha \in \Pi$ and the result is obviously true. Now assume $\operatorname{ht}(\alpha)>1$. Let $\xi: \alpha_{1}=\alpha, \alpha_{2}, \ldots, \alpha_{r}$ be a root path from $\alpha$ to $\Pi$. Then $\xi^{\prime}: \alpha_{2}, \alpha_{3}, \ldots, \alpha_{r}$ is a root path from $\alpha_{2}$ to $\Pi$ with $\operatorname{ht}\left(\alpha_{2}\right)<\operatorname{ht}(\alpha)$ and $\alpha_{2}=s_{\delta}(\alpha)$ for some $\delta \in \Pi$. Note that $\alpha_{2}$ is a long root of $\Phi$. Write

$$
\alpha_{2}=\sum_{\beta \in \Pi} a_{\beta}^{\prime} \beta, \quad a_{\beta}^{\prime} \in \mathbb{Z} .
$$

Then by inductive hypothesis, we have

$$
h\left(\alpha_{2}, \xi^{\prime}\right)=\sum_{\beta \in \Pi} \frac{|\beta|^{2}}{\left|\alpha_{2}\right|^{2}} a_{\beta}^{\prime} .
$$

Since $\left\langle\alpha, \delta^{\vee}\right\rangle=|\alpha|^{2} /|\delta|^{2}$ by the assumption $s_{\delta}(\alpha)<\alpha$, we have

$$
\alpha=\alpha_{2}+\frac{|\alpha|^{2}}{|\delta|^{2}} \delta=\sum_{\substack{\beta \in \Pi \\ \beta \neq \delta}} a_{\beta}^{\prime} \beta+\left(a_{\delta}^{\prime}+\frac{|\alpha|^{2}}{|\delta|^{2}}\right) \delta .
$$

This implies that

$$
\begin{aligned}
h(\alpha, \xi) & =h\left(\alpha_{2}, \xi^{\prime}\right)+1=\sum_{\beta \in \Pi} \frac{|\beta|^{2}}{\left|\alpha_{2}\right|^{2}} a_{\beta}^{\prime}+1 \\
& =\sum_{\substack{\beta \in \Pi \\
\beta \neq \delta}} \frac{|\beta|^{2}}{\left|\alpha_{2}\right|^{2}} a_{\beta}^{\prime}+\frac{|\delta|^{2}}{\left|\alpha_{2}\right|^{2}}\left(a_{\delta}^{\prime}+\frac{\left|\alpha_{2}\right|^{2}}{|\delta|^{2}}\right)=\sum_{\beta \in \Pi} \frac{|\beta|^{2}}{|\alpha|^{2}} a_{\beta}
\end{aligned}
$$

by noting $|\alpha|=\left|\alpha_{2}\right|$. 
We see from Lemma 1.1 that, for any $\alpha \in \Phi^{+}$, the length of a root path $\xi$ from $\alpha$ to $\Pi$ is only dependent on $\alpha$ but not on the choice of the path $\xi$. So we can denote $h(\alpha, \xi)$ simply by $h(\alpha)$.

Let $\Phi^{\vee}$ be the dual root system of $\Phi$, i.e. $\Phi^{\vee}=\left\{\alpha^{\vee} \mid \alpha \in \Phi\right\}$. Then $\Pi^{\vee}=\left\{\alpha^{\vee} \mid \alpha \in \Pi\right\}$ and $\left(\Phi^{\vee}\right)^{+}=\left\{\alpha^{\vee} \mid \alpha \in \Phi^{+}\right\}$are a simple root system and the corresponding positive root system of $\Phi^{\vee}$, respectively. We can define the number $h^{\vee}\left(\alpha^{\vee}\right)$ for any $\alpha^{\vee} \in\left(\Phi^{\vee}\right)^{+}$in the same way as that for a root of $\Phi$. That is, $h^{\vee}\left(\alpha^{\vee}\right)$ is the length of a root path from $\alpha^{\vee}$ to $\Pi^{\vee}$ in $\left(\Phi^{\vee}\right)^{+}$.

LEMMA 1.2. For any $\alpha \in \Phi^{+}$, we have $h(\alpha)=h^{\vee}\left(\alpha^{\vee}\right)$.

Proof. For any $\delta \in \Pi$, we have the following equivalence.

$$
s_{\delta}(\alpha)<\alpha \Leftrightarrow\left\langle\alpha, \delta^{\vee}\right\rangle>0 \Leftrightarrow\left\langle\alpha^{\vee}, \delta\right\rangle>0 \Leftrightarrow s_{\delta^{\vee}}\left(\alpha^{\vee}\right)<\alpha^{\vee} .
$$

Apply induction on $h(\alpha) \geq 1$. When $h(\alpha)=1$, we have $\alpha \in \Pi$ and hence $\alpha^{\vee} \in \Pi^{\vee}$. So $h^{\vee}\left(\alpha^{\vee}\right)=1$, and the result is true in this case. Now assume $h(\alpha)>1$. Then there exists some $\delta \in \Pi$ with $\left\langle\alpha, \delta^{\vee}\right\rangle>0$. So $h\left(s_{\delta}(\alpha)\right)=h(\alpha)-1$. By inductive hypothesis, we have

$$
h\left(s_{\delta}(\alpha)\right)=h^{\vee}\left(\left(s_{\delta}(\alpha)\right)^{\vee}\right)=h^{\vee}\left(s_{\delta}^{\vee}\left(\alpha^{\vee}\right)\right) .
$$

But by (1), we have

$$
h^{\vee}\left(s_{\delta^{\vee}}\left(\alpha^{\vee}\right)\right)=h^{\vee}\left(\alpha^{\vee}\right)-1 .
$$

Thus we get $h(\alpha)=h^{\vee}\left(\alpha^{\vee}\right)$.

2. The number $d(\alpha)$. We shall deduce a formula for the number $d(\alpha)$ for any $\alpha \in \Phi^{+}$.

For $\alpha, \beta \in \Phi$, we call all roots of the form $\alpha+i \beta \quad(i \in \mathbb{Z})$ the $\beta$-string through $\alpha$. Let $\alpha \in \Phi^{+}$and $\delta \in \Pi$ satisfy the inequality $\left\langle\alpha, \delta^{\vee}\right\rangle>0$. Then it is easily seen that $\alpha, \alpha-\delta, \ldots, \alpha-\left\langle\alpha, \delta^{\vee}\right\rangle \delta$ is the $\delta$-string through $\alpha$ except for the case when $\alpha$ is the highest short root of the root system of type $G_{2}$.

Lemma 2.1. Given $\alpha \in \Phi^{+}$and $\delta \in \Pi$ with $\left\langle\alpha, \delta^{\vee}\right\rangle>0$. Let $\alpha^{\prime}=s_{\delta}(\alpha)$. Then (i) $D\left(\alpha^{\prime}\right)=s_{\delta}(D(\alpha))$.

(ii) $s_{\delta}\left(D^{+}\left(\alpha^{\prime}\right)\right)=D^{+}(\alpha) \cup\{-\delta\}$, provided that $\alpha$ is not the highest short root of the root system of type $G_{2}$;

(iii) $d\left(\alpha^{\prime}\right)=d(\alpha)+1$ under the same assumption as that in (ii). 
Proof. (i) $\beta \in D\left(\alpha^{\prime}\right) \Leftrightarrow \beta+\alpha^{\prime} \in \Phi \Leftrightarrow s_{\delta}\left(s_{\delta}(\beta)+\alpha\right) \in \Phi \Leftrightarrow s_{\delta}(\beta)+\alpha \in$ $\Phi \Leftrightarrow s_{\delta}(\beta) \in D(\alpha) \Leftrightarrow \beta \in s_{\delta}(D(\alpha))$.

(ii) First we shall show $s_{\delta}\left(D^{+}(\alpha)\right) \subset D^{+}\left(\alpha^{\prime}\right)$. Let $\beta \in s_{\delta}\left(D^{+}(\alpha)\right)$. Then $\beta \in D\left(\alpha^{\prime}\right)$ by (i). If $\beta \in D^{-}\left(\alpha^{\prime}\right) \subseteq \Phi^{-}$, then by the fact $s_{\delta}(\beta) \in D^{+}(\alpha) \subseteq \Phi^{+}$, we have $\beta=-\delta$. Since $\alpha, \alpha-\delta, \ldots, \alpha-$ $\left\langle\alpha, \delta^{\vee}\right\rangle \delta$ is the $\delta$-string through $\alpha$ by the above remark, we see that $\alpha+s_{\delta}(\beta)=\alpha+\delta \notin \Phi$ which contradicts the condition $s_{\delta}(\beta) \in D^{+}(\alpha)$. Thus we have $\beta \in D^{+}\left(\alpha^{\prime}\right)$ and so $s_{\delta}\left(D^{+}(\alpha)\right) \subset D^{+}\left(\alpha^{\prime}\right)$, i.e. $D^{+}(\alpha) \subset$ $s_{\delta}\left(D^{+}\left(\alpha^{\prime}\right)\right)$.

It is obvious that $\{-\delta\} \subseteq s_{\delta}\left(D^{+}\left(\alpha^{\prime}\right)\right)$. Thus it remains to show the reversing inclusion. Now assume $\beta \in s_{\delta}\left(D^{+}\left(\alpha^{\prime}\right)\right)$. Then $s_{\delta}(\beta) \in$ $D^{+}\left(\alpha^{\prime}\right)$. This implies that $s_{\delta}(\beta)+\alpha^{\prime} \in \Phi$ and $s_{\delta}(\beta) \in \Phi^{+}$. Hence $\beta+\alpha \in \Phi$ and $s_{\delta}(\beta) \in \Phi^{+}$. But then we have either $\beta \in D^{+}(\alpha)$ or $\beta=-\delta$, which implies $s_{\delta}\left(D^{+}\left(\alpha^{\prime}\right)\right) \subseteq D^{+}(\alpha) \cup\{-\delta\}$.

(iii) This is an immediate consequence of (ii).

REMARK. In the case when the type of $\Phi$ is $G_{2}$, let $\Pi=\{\gamma, \delta\}$ with $\delta$ short. Then $D^{+}(2 \delta+\gamma)=\{\delta, \delta+\gamma\}, D^{+}(\delta+\gamma)=\{\delta, 2 \delta+\gamma\}$ and $\delta+\gamma=s_{\delta}(2 \delta+\gamma)$. Thus the results (ii), (iii) of Lemma 2.1 do not hold in this case.

In $\Phi^{+}$, let $\alpha^{l}$ be the highest long root and let $\alpha^{s}$ be the highest short root, where we stipulate $\alpha^{s}=\alpha^{l}$ in the case when all the roots of $\Phi$ have the same length.

THEOREM 2.2. Given $\alpha \in \Phi^{+}$.

(i) If $\alpha$ is short and if the type of $\Phi$ is not $G_{2}$, then

$$
h(\alpha)+d(\alpha)=\operatorname{ht}\left(\alpha^{l}\right) .
$$

(ii) If $\alpha$ is long, then

$$
h(\alpha)+d(\alpha)=\operatorname{ht}\left(\alpha^{s}\right)
$$

Proof. First assume that the result has been shown to be true in the case when $\alpha=\alpha^{s}$ in (i) and $\alpha=\alpha^{l}$ in (ii). Apply reversing induction on $h(\alpha) \leq h\left(\alpha^{s}\right)$ in (i) and on $h(\alpha) \leq h\left(\alpha^{l}\right)$ in (ii). Now assume that $\alpha$ is either short with $h(\alpha)<h\left(\alpha^{s}\right)$ or long with $h(\alpha)<h\left(\alpha^{l}\right)$. Then there must exist some $\delta \in \Pi$ with $\left\langle\alpha, \delta^{\vee}\right\rangle<0$. So $\alpha^{\prime}=s_{\delta}(\alpha)>\alpha$ with $h\left(\alpha^{\prime}\right)=h(\alpha)+1$. We see $\left\langle\alpha^{\prime}, \delta^{\vee}\right\rangle>0$. By Lemma 2.1(iii), we 
have $d\left(\alpha^{\prime}\right)=d(\alpha)-1$. So by inductive hypothesis, we get

$$
\begin{aligned}
h(\alpha)+d(\alpha) & =\left(h\left(\alpha^{\prime}\right)-1\right)+\left(d\left(\alpha^{\prime}\right)+1\right) \\
& =h\left(\alpha^{\prime}\right)+d\left(\alpha^{\prime}\right) \\
& = \begin{cases}\operatorname{ht}\left(\alpha^{l}\right) & \text { if } \alpha \text { is short, } \\
\operatorname{ht}\left(\alpha^{s}\right) & \text { if } \alpha \text { is long, }\end{cases}
\end{aligned}
$$

by noting $|\alpha|=\left|\alpha^{\prime}\right|$.

Thus it remains to show that assertion (i) is true for $\alpha=\alpha^{s}$ and that assertion (ii) is true for $\alpha=\alpha^{l}$.

In the case when the Dynkin diagram is simply laced, we have $h\left(\alpha^{S}\right)=h t\left(\alpha^{S}\right)$ by Lemma 1.1(i). Clearly, $d\left(\alpha^{S}\right)=0$. So our result is true in this case. Now assume that $\Phi$ contains roots of two different lengths. If $\Phi$ has type $B_{n}$, then $h\left(\alpha^{s}\right)=n, d\left(\alpha^{s}\right)=n-1$, $\operatorname{ht}\left(\alpha^{l}\right)=2 n-1, d\left(\alpha^{l}\right)=0$ and $h\left(\alpha^{l}\right)=h^{\vee}\left(\left(\alpha^{l}\right)^{\vee}\right)=\operatorname{ht}\left(\left(\alpha^{l}\right)^{\vee}\right)=$ $\operatorname{ht}\left(\alpha^{s}\right)=2 n-2$ by Lemmas 1.2 and 1.1(i). If $\Phi$ has type $C_{n}$, then $h\left(\alpha^{s}\right)=2 n-2, d\left(\alpha^{s}\right)=1, \operatorname{ht}\left(\alpha^{l}\right)=2 n-1$ and $d\left(\alpha^{l}\right)=0$. We also have

$$
h\left(\alpha^{l}\right)=h^{\vee}\left(\left(\alpha^{l}\right)^{\vee}\right)=\operatorname{ht}\left(\left(\alpha^{l}\right)^{\vee}\right)=\operatorname{ht}\left(\alpha^{s}\right)=n
$$

by Lemmas 1.2 and 1.1(i). If $\Phi$ has type $F_{4}$, then $h\left(\alpha^{s}\right)=8, d\left(\alpha^{s}\right)=$ $3, \operatorname{ht}\left(\alpha^{l}\right)=11$ and $d\left(\alpha^{l}\right)=0$. By the same reason as above, we have

$$
h\left(\alpha^{l}\right)=h^{\vee}\left(\left(\alpha^{l}\right)^{\vee}\right)=\operatorname{ht}\left(\left(\alpha^{l}\right)^{\vee}\right)=\operatorname{ht}\left(\alpha^{s}\right)=8 .
$$

If $\Phi$ has type $G_{2}$, then $d\left(\alpha^{l}\right)=0$ and $h\left(\alpha^{l}\right)=h t\left(\alpha^{s}\right)=3$. Thus in all the cases, our result is true.

Corollary 2.3. Assume that the type of $\Phi$ is not $G_{2}$. Then for any short root $\alpha$ of $\Phi^{+}$, we have the equation

$$
\operatorname{ht}(\alpha)+d(\alpha)=h-1,
$$

where $h$ is the Coxeter number of $\Phi$.

Proof. We have $h(\alpha)=\operatorname{ht}(\alpha)$ by Lemma 1.1(i). Since $\operatorname{ht}\left(\alpha^{l}\right)=$ $h-1$, our result follows immediately from Theorem 2.2(i).

3. The number of certain rank 2 subsystems in $\Phi$. Let $g(\Phi)$ be the number of subsystems of $\Phi$ of rank 2 and of type other than $A_{1} \times A_{1}$. Then $g(\Phi)$ is also equal to the number of positive subsystems of $\Phi^{+}$ of rank 2 and of type $\neq A_{1} \times A_{1}$. In this section, we shall compute the number $g(\Phi)$ for $\Phi$ of any type. 
LEMMA 3.1. If the Dynkin diagram of $\Phi$ is simply laced, then

$$
g(\Phi)=\frac{1}{2} \sum_{\alpha \in \Phi^{+}} d(\alpha) \text {. }
$$

Proof. Under our assumption, the only possible type for a subsystem of $\Phi^{+}$of rank 2 and of type $\neq A_{1} \times A_{1}$ is $A_{2}$. Each of such subsystems could be obtained by first taking a root $\alpha \in \Phi^{+}$and then taking any root $\beta$ in the set $D^{+}(\alpha)$ to form a subsystem $\{\alpha, \beta, \alpha+\beta\}$. Since such a subsystem is obtained twice in the above way, this implies the required formula (3) for the number $g(\Phi)$.

Define

$$
\begin{gathered}
H(\Phi)=\sum_{\alpha \in \Phi^{+}} \mathrm{ht}(\alpha), \quad H^{s}(\Phi)=\sum_{\substack{\alpha \in \Phi^{+} \\
\text {short }}} \mathrm{ht}(\alpha) \text { and } \\
H^{l}(\Phi)=\sum_{\substack{\alpha \in \Phi^{+} \\
\text {long }}} \mathrm{ht}(\alpha) .
\end{gathered}
$$

These numbers could be computed for any irreducible root system $\Phi$. Define $\left(\begin{array}{c}m \\ n\end{array}\right)=\frac{m !}{n !(m-n) !}$ for any integers $m, n, 0 \leq n \leq m$.

LEMMA 3.2.

\begin{tabular}{|l|c|c|c|}
\hline Type of $\Phi$ & $H(\Phi)$ & $H^{s}(\Phi)$ & $H^{l}(\Phi)$ \\
\hline$A_{n}(n \geq 1)$ & $\left(\begin{array}{c}n+2 \\
3\end{array}\right)$ & & \\
\hline$B_{n}(n \geq 2)$ & $\frac{n(n+1)(4 n-1)}{6}$ & $\left(\begin{array}{c}n+1 \\
2\end{array}\right)$ & $4\left(\begin{array}{c}n+1 \\
3\end{array}\right)$ \\
\hline$C_{n}(n \geq 2)$ & $\frac{n(n+1)(4 n-1)}{6}$ & $\frac{n(n-1)(4 n+1)}{6}$ & $n^{2}$ \\
\hline$D_{n}(n \geq 4)$ & $\frac{n(n-1)(2 n-1)}{3}$ & & \\
\hline$E_{6}$ & 156 & & \\
\hline$E_{7}$ & 399 & & \\
\hline$E_{8}$ & 1240 & & 64 \\
\hline$F_{4}$ & 110 & 46 & 10 \\
\hline$G_{2}$ & 16 & 6 & \\
\hline
\end{tabular}


Now we can compute the numbers $g(\Phi)$ for $\Phi$ of types $A_{n}, n \geq 1$, $D_{m}, m \geq 4$, and $E_{i}, i=6,7,8$ as follows.

THEOREM 3.3 .

\begin{tabular}{|l|c|}
\hline Type of $\Phi$ & $g(\Phi)$ \\
\hline$A_{n}(n \geq 1)$ & $\left(\begin{array}{c}n+1 \\
3\end{array}\right)$ \\
\hline$D_{n}(n \geq 4)$ & $4\left(\begin{array}{c}n \\
3\end{array}\right)$ \\
\hline$E_{6}$ & 120 \\
\hline$E_{7}$ & 336 \\
\hline$E_{8}$ & 1120 \\
\hline
\end{tabular}

Proof. By Corollary 2.3 and Lemma 3.1, we have

$$
\begin{aligned}
g(\Phi) & =\frac{1}{2} \sum_{\alpha \in \Phi^{+}} d(\alpha)=\frac{1}{2} \sum_{\alpha \in \Phi^{+}}(h-1-\operatorname{ht}(\alpha)) \\
& =\frac{1}{2}\left((h-1)\left|\Phi^{+}\right|-H(\Phi)\right) .
\end{aligned}
$$

Thus we have $g\left(\Phi\left(A_{n}\right)\right)=\frac{1}{2}\left(n\left(\begin{array}{c}n+1 \\ 2\end{array}\right)-\left(\begin{array}{c}n+2 \\ 3\end{array}\right)\right)=\left(\begin{array}{c}n+1 \\ 3\end{array}\right)$ for $n \geq 1$. For $n \geq 4$, we have

$$
g\left(\Phi\left(D_{n}\right)\right)=\frac{1}{2}\left((2 n-3) n(n-1)-\frac{n(n-1)(2 n-1)}{3}\right)=4\left(\begin{array}{l}
n \\
3
\end{array}\right) .
$$

Also, we have $g\left(\Phi\left(E_{6}\right)\right)=\frac{1}{2}(11 \cdot 36-156)=120$,

$$
g\left(\Phi\left(E_{7}\right)\right)=\frac{1}{2}(17 \cdot 63-399)=336,
$$

and $g\left(\Phi\left(E_{8}\right)\right)=\frac{1}{2}(29 \cdot 120-1240)=1120$.

Now assume that $\Phi$ contains roots of two different lengths and that the type of $\Phi$ is not $G_{2}$. Then the possible types for a subsystem $\Phi^{\prime}$ of $\Phi$ of rank 2 and of type $\neq A_{1} \times A_{1}$ are $A_{2}$ and $B_{2}$. Let $u(\Phi)$ be the cardinality of the set

$\left\{\{\alpha, \beta\} \mid \alpha, \beta \in \Phi^{+}\right.$have different lengths with $\left.\alpha+\beta \in \Phi^{+}\right\}$.

Then it is easily seen that the following formula for $g(\Phi)$ holds.

$$
g(\Phi)=\frac{1}{2} \sum_{\alpha \in \Phi^{+}} d(\alpha)-u(\Phi)
$$


First let us consider the case when $\Phi$ has type $C_{n}, n \geq 2$. We see that a subsystem $\Phi^{\prime}$ of $\Phi$ has type $A_{2}$ only if all the roots in $\Phi^{\prime}$ are short. This implies that for each long root $\beta \in \Phi^{+}$, the set $D^{+}(\beta)$ contains no long root and hence $u(\Phi)=\sum_{\beta \in \Phi^{+} \text {long }} d(\beta)$. So by (4), we get

$$
\begin{aligned}
g(\boldsymbol{\Phi}) & =\frac{1}{2} \sum_{\alpha \in \Phi^{+}} d(\alpha)-\sum_{\substack{\beta \in \Phi^{+} \\
\text {long }}} d(\beta)=\frac{1}{2}\left(\sum_{\substack{\alpha \in \Phi^{+} \\
\text {short }}} d(\alpha)-\sum_{\substack{\beta \in \Phi^{+} \\
\text {long }}} d(\beta)\right) \\
& =\frac{1}{2}\left(\sum_{\substack{\alpha \in \Phi^{+} \\
\text {short }}}(h-1-\mathrm{ht}(\alpha))-\sum_{i=1}^{n}(i-1)\right)
\end{aligned}
$$

by Theorem 2.2, Corollary 2.3 and Lemma 1.2 . Then by Lemma 3.2, we have

$$
\begin{aligned}
g(\Phi) & =\frac{1}{2}\left((2 n-1) n(n-1)-\frac{n(n-1)(4 n+1)}{6}-\frac{n(n-1)}{2}\right) \\
& =\frac{n(n-1)(4 n-5)}{6} .
\end{aligned}
$$

Since the root system of type $B_{n}$ is the dual of the one of type $C_{n}$, there exists a bijection from the set of subsystems of the root system of type $C_{n}$ to that of type $B_{n}$ by sending $\Phi^{\prime}$ to $\Phi^{\prime}{ }^{\prime}$. Such a bijective map preserves the ranks of subsystems and also preserves the types of them whenever their ranks are not greater than 2 . This implies that we also have $g(\Phi)=\frac{n(n-1)(4 n-5)}{6}$ when $\Phi$ has type $B_{n}$.

Next assume that $\Phi$ has type $F_{4}$. By Theorem 2.2, Lemma 3.2 and Lemmas $1.1,1.2$, we get

$$
\begin{aligned}
\frac{1}{2} \sum_{\alpha \in \Phi^{+}} d(\alpha) & =\frac{1}{2}\left(\sum_{\substack{\alpha \in \Phi^{+} \\
\text {short }}}\left(\mathrm{ht}\left(\alpha^{l}\right)-\mathrm{ht}(\alpha)\right)+\sum_{\substack{\beta \in \Phi^{+} \\
\text {long }}}\left(\mathrm{ht}\left(\alpha^{s}\right)-\mathrm{ht}\left(\beta^{\vee}\right)\right)\right) \\
& =\frac{1}{2}\left(\frac{1}{2}\left|\Phi^{+}\right|\left(\mathrm{ht}\left(\alpha^{l}\right)+\mathrm{ht}\left(\alpha^{s}\right)\right)-2 H^{s}(\Phi)\right) \\
& =\frac{1}{2}\left(\frac{1}{2} \cdot 24 \cdot(11+8)-92\right) \\
& =68 .
\end{aligned}
$$

Also, by a direct computation, we get $u(\Phi)=18$. So by (4), we have

$$
g(\Phi)=68-18=50 \text {. }
$$


Finally, it is easily seen that $g(\Phi)=3$ when $\Phi$ has type $G_{2}$. Summing up, we get the following table.

THEOREM 3.4.

\begin{tabular}{|l|c|}
\hline Type of $\Phi$ & $g(\Phi)$ \\
\hline$B_{n}$ or $C_{n}(n \geq 2)$ & $\frac{n(n-1)(4 n-5)}{6}$ \\
\hline$F_{4}$ & 50 \\
\hline$G_{2}$ & 3 \\
\hline
\end{tabular}

From the above discussion, we can deduce even more precise conclusion. We note that in any irreducible root system $\Phi$, there exist at most two different types of subsystems which have rank 2 and types $\neq A_{1} \times A_{1}$. Let $g^{\prime}(\Phi)$ be the number of subsystems of $\Phi$ of type $A_{2}$ and let $g^{\prime \prime}(\Phi)$ be the number of subsystems of $\Phi$ of type $B_{2}$ or $G_{2}$. Then by Theorem 3.3, we have

$$
g^{\prime}\left(\Phi\left(B_{n}\right)\right)=g^{\prime}\left(\Phi\left(C_{n}\right)\right)=g\left(\Phi\left(D_{n}\right)\right)=4\left(\begin{array}{l}
n \\
3
\end{array}\right) \quad \text { for } n \geq 4
$$

by noting that all the long (resp. short) roots of $\Phi\left(B_{n}\right)$ (resp. $\Phi\left(C_{n}\right)$ ) form a root system of type $D_{n}$. Hence we also have

$$
\begin{aligned}
g^{\prime \prime}\left(\Phi\left(B_{n}\right)\right) & =g^{\prime \prime}\left(\Phi\left(C_{n}\right)\right)=g\left(\Phi\left(B_{n}\right)\right)-g^{\prime}\left(\Phi\left(B_{n}\right)\right) \\
& =\frac{n(n-1)(4 n-5)}{6}-4\left(\begin{array}{l}
n \\
3
\end{array}\right) \\
& =\left(\begin{array}{l}
n \\
2
\end{array}\right) .
\end{aligned}
$$

On the other hand, we have

$$
\begin{aligned}
g^{\prime \prime}\left(\Phi\left(F_{4}\right)\right) & =u\left(\Phi\left(F_{4}\right)\right)=18 \text { and } \\
g^{\prime}\left(\Phi\left(F_{4}\right)\right) & =g\left(\Phi\left(F_{4}\right)\right)-g^{\prime \prime}\left(\Phi\left(F_{4}\right)\right)=50-18=32
\end{aligned}
$$

Finally, it is obvious that $g^{\prime}\left(\Phi\left(G_{2}\right)\right)=2$ and $g^{\prime \prime}\left(\Phi\left(G_{2}\right)\right)=1$. Summing up, we have the following table. 
THEOREM 3.5.

\begin{tabular}{|l|c|c|}
\hline Type of $\Phi$ & $g^{\prime}(\Phi)$ & $g^{\prime \prime}(\Phi)$ \\
\hline$B_{n}, C_{n}(n \geq 2)$ & $4\left(\begin{array}{l}n \\
3\end{array}\right)$ & $\left(\begin{array}{l}n \\
2\end{array}\right)$ \\
\hline$F_{4}$ & 32 & 18 \\
\hline$G_{2}$ & 2 & 1 \\
\hline
\end{tabular}

Proof. By the above discussion, it remains to show the result for $\Phi$ being of types $B_{m}$ or $C_{m}, m=2,3$. But this could be checked directly.

\section{REFERENCES}

[1] N. Bourbaki, Groupes et Algèbres de Lie, Ch. 4-6, Hermann, Paris, 1968.

[2] Jian-yi Shi, Alcoves corresponding to an affine Weyl group, J. London Math. Soc., 35 (1987), 42-55.

[3] __ Sign types corresponding to an affine Weyl group, J. London Math. Soc., 35 (1987), 56-74.

Received November 15, 1991. Supported by the National Science Foundation of China and by the Science Foundation of the University Doctoral Program of CNEC.

East China Normal University 3663 ZHONGSHAN ROAD (NORTHERN)

Shanghai 200062, China 


\section{CONTENTS}

G. D. Anderson, M. K. Vamanamurthy, and M. Vuorinen, Inequalities for quasi-

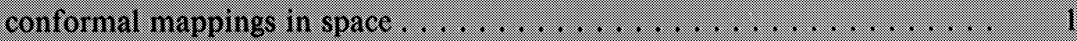

T. Bhattacharya, A nonexistence result for the $n$-Laplacian . . . . . . . . . . . . 19

J. A. Cima, K. Stroethoff, and K. Yale, Bourgain algebras on the unit disk . . . . . 27

J. A. Fridy and C. Orhan, Lacunary statistical convergence . . . . . . . . . . . . . . 43

D. Grenier, On the shape of fundamental domains in $\mathrm{GL}(n, \mathbf{R}) / \mathrm{O}(n) \ldots \ldots . . .53$

B. Jiang and J. Guo, Fixed points of surface diffeomorphisms . . . . . . . . . . . 67

P. Lejarraga, The moduli of rational Weierstrass fibrations over $\mathbf{P}^{\prime}$ : singularities 91

G. J. Martin, On discrete isometry groups of negative curvature . . . . . . . . . . 109

T. Nakashima, Adjoint linear systems on a surface of general type in positive characteristic . . . . . . . . . . . . . . . . . . . . . . . . . 129

B. Ralph, A homotopy transfer for finite group actions $\ldots \ldots \ldots \ldots \ldots \ldots \ldots$

Y. Rong, Maps between Seifert fibered spaces of infinite $\pi_{1} \ldots \ldots \ldots \ldots \ldots, 143$

J.-Y. Shi, Some numeric results on root systems . . . . . . . . . . . . 155

E. Spanier, Singular homology and cohomology with local coefficients and duality

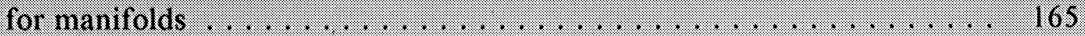




\section{PACIFIC JOURNAL OF MATHEMATICS}

Volume $160 \quad$ No. $1 \quad$ September 1993

Inequalities for quasiconformal mappings in space

GLEN DOUglas ANDERson, MaVina KRISHNa VAMANAMURThy and MATTI VUORINEN

A nonexistence result for the $n$-Laplacian

TILAK BHATTACHARYA

Bourgain algebras on the unit disk

Joseph A. Cima, Karel M. Stroethoff and Keith Yale

Lacunary statistical convergence

JOHN ALBERT FRIDY and CIHAN ORHAN

On the shape of fundamental domains in $\operatorname{GL}(n, \mathbf{R}) / \mathrm{O}(n)$

DOUGLAS MARTIN GRENIER

Fixed points of surface diffeomorphisms

BOJU JIANG and JIANHAN GUO

The moduli of rational Weierstrass fibrations over $\mathbf{P}^{1}$ : singularities

PABLO LEJARRAGA

On discrete isometry groups of negative curvature

GAVEN MARTIN

Adjoint linear systems on a surface of general type in positive characteristic

TOHRU NAKASHIMA

A homotopy transfer for finite group actions

WILLIAM J. RALPH

Maps between Seifert fibered spaces of infinite $\pi_{1}$

YONGWU RONG

Some numeric results on root systems

J. Y. SHI

Singular homology and cohomology with local coefficients and duality for manifolds

EDWIN SPANIER 